\title{
Zigbee-based Local Army Strategy Network Configurations for Multimedia Military Service
}

\author{
Seung-Mo Je ${ }^{1 *}$
}

\begin{abstract}
With the rapid evolution of communication technology, it became possible to overcome the spatial and temporal limitations faced by humans to some extent. Furthermore, the quality of personal life was revolutionized with the emergence of the personal communication device commonly known as the smart phone. In terms of defense networks, however, due to restrictions from the military and security perspectives, the use of smart phones has been prohibited and controlled in the army; thus, they are not being used for any defense strategy purposes as yet. Despite the current consideration of smart phones for military communication, due to the difficulties of network configuration and the high cost of the necessary communication devices, the main tools of communication between soldiers are limited to the use of flag, voice or hand signals, which are all very primitive. Although these primitive tools can be very effective in certain cases, they cannot overcome temporal and spatial limitations. Likewise, depending on the level of the communication skills of each individual, communication efficiency can vary significantly. As the term of military service continues to be shortened, however, types of communication of varying efficiency depending on the levels of skills of each individual newly added to the military is not desirable at all. To address this problem, it is essential to prepare an intuitive network configuration that facilitates use by soldiers in a short period of time by easily configuring the strategy network at a low cost while maintaining its security. Therefore, in this article, the author proposes a Zigbee-based local strategic network by using Opnet and performs a simulation accordingly.
\end{abstract}

Key Words: Game Theory, IoT, Tactical Network, Mesh Network, OPNET, Multimedia Military Service.

\section{Introduction}

The army's strategy network is currently configured to support efficient use of the given limited resources. Under the current defense system of Korea, however, communication is (only) allowed at the lowest level, i.e. between squad commanders, with the result that soldiers cannot be deployed or used efficiently [1-3]. Communication between soldiers in a platoon or between platoon commanders mostly relies on voice or hand signals or other primitive communication methods, which nonetheless can be highly effective in some situations [4-5]. First, no costs are incurred other than those for setting a signal communicated between them. This means that communication between them is possible even without using additional communication tools. Second, it leads to highly flexible network configuration, for which a certain level of agreement should be reached between the parties concerned; other than that, however, there is no need to pay for the installation of lines or antennae. Without additional communication tools, soldiers can communicate with each other and send commands. Third, the security required for strategic operation can be achieved almost fully without any additional efforts, despite the temporal and spatial limitations inherent to these primitive signals.

\section{Military Resource Status}

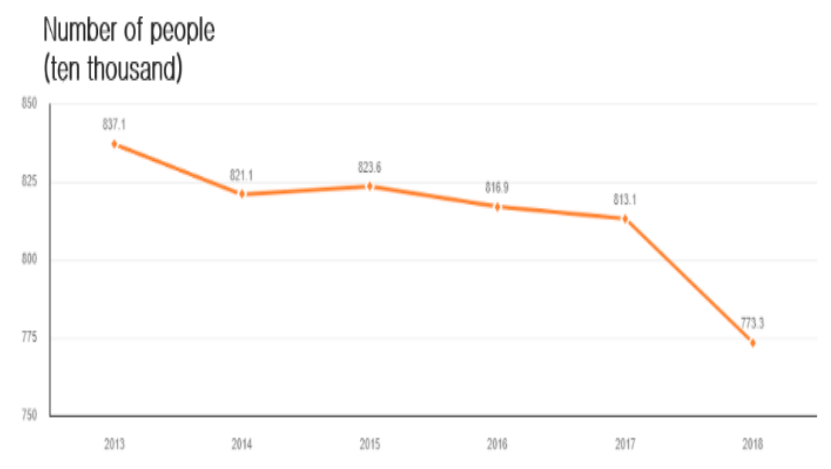

Fig. 1. Military Resource Status [4].

Manuscript received September 12, 2019; Revised September 26, 2019; Accepted September 27, 2019. (ID No. JMIS-19M-09-027) Corresponding Author (*): Seung-Mo Je, Department of Computer Science Education, Korea University, Seongbuk-gu, Seoul, 02841, Republic of Korea, jsm3316@korea.ac.kr or jsm3316@naver.com

${ }^{1}$ Seung-Mo Je, Department of Computer Science Education, Korea University, Seongbuk-gu, Seoul, 02841, Republic of Korea, jsm3316@korea.ac.kr or jsm3316@naver.com 
Nonetheless, Korea's rapidly declining birthrate, dwindling military human resources, and shorter term of military service have given rise to the problem of an absolute insufficiency of military manpower, as high skills are required, including a considerable decline of the efficiency of primitive communications reliant on the skills of soldiers [6-7]. In addition, as the total pool of human resources available to the military decreases, the strategic value of each soldier increases accordingly. Moreover, the temporal and spatial limitations of primitive communication methods with security advantages can be seen as a double-edged sword; thus, it can restrict the level of communication to a certain degree and thus hinder smooth communication, and must be supplemented by the use, albeit inefficient, of military human resources. This factor has given rise to the need to improve the low efficiency of primitive communication methods while maintaining their advantages related to the operation of military human resources.

\section{Related Research}

\subsection{OPNET Modeler (Riverbed Modeler)}

The origin of OPNET Modeler and Riverbed Modeler, which are getting the spotlight as the best communications and networks simulation solutions, can be trace back the network simulator carried forward as part of the project which was ordered by the Department of Defense to MIT in 1986 and have reached their present forms as a result of continuous efforts and studies by the participating developers. OPNET solutions have been used and verified over $20+$ years, and with the established trust, they are expanding their scope of application to the areas of Network Capacity Planning, Network Engineering, Network Management, etc [3].

\subsection{Zigbee Characteristics}

Zigbee is a Wi-Fi network protocol characterized by its low cost, low data transmission speed, short transmission distance, and high battery efficiency. IEEE 802.15.4 defines this Wi-Fi network standard by characterizing the physical layers and the MAC layers [8-9]. The Zigbee Alliance and the IEEE 802.15 WPAN Working group successfully implemented the source. It supports bandwidths of $868 \mathrm{MHz}, 915 \mathrm{MHz}$, and $2.4 \mathrm{GHz}$, and a data transmission speed of $250 \mathrm{Kbit}$ per sec. It also has a power saving mode, enabling the battery to last for several years. In addition, Zigbee supports the mesh network [10-11].

\subsection{Mesh Network}

In Zigbee, each node performs the role of a Wi-Fi base station. Even if one node is disconnected, the other nodes can still communicate with each other; and when the entire network is down, a local network can be formed. These characteristics of the mesh network enable users to exchange data by forming a local network in environments where it is very difficult to configure a network, such as disaster-stricken or isolated locations [12-14].

In addition, it can form an additional network, so there is no need to pay a fee to the network base station for the frequency band. The traffic is distributed all over, without focusing on a certain base station, thereby improving network stability and easing the dependence on a specific base station. Since there is also a group that manages base stations, monopoly by a specific company may be prevented [15-20].

\subsection{UWB communication terminal for soldiers}

Among the existing studies, there was a research work focusing on the implementation of a squad unit communication based on voice data transmission using a UWB communication terminal for soldiers [21-25]. However, in a tactical environment where no communication infrastructures are available, such a method has a disadvantage of not being portable as the size of the terminal is too big. Aiming to exchange signals between Zigbee nodes, this study proposed a method of using a Zigbee communication network as a tactical network by reducing the size of the terminal nodes which could be used for long hours without recharging.

\section{Primitive Communication Improvement Plan: Use of Zigbee in the Army Strategy Network}

To protect the country effectively with limited military defense resources, efficient use of the given resources is crucial. If the inefficiency associated with the deployment and operations of soldiers can be improved in a way that relies on primitive communication methods, the author expects various advantages in defending the nation. Fig. 2 illustrates an OPNET simulation-based methodology for linking the existing army defense network to the squad strategy network.

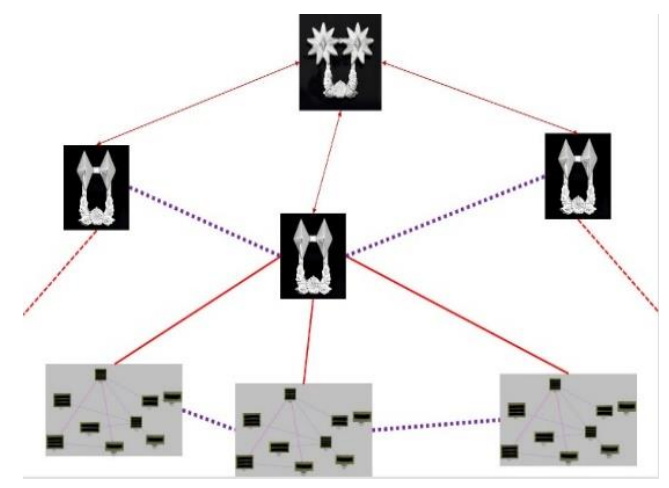

Fig. 2. OPNET Simulation-Based Methodology for Linking the Existing Army Strategy Network with the Squad Strategy Network. 
Since Zigbee is a low-cost Wi-Fi communication protocol, it is possible to distribute communication devices to each individual soldier and to minimize the huge losses associated with the primitive communication methods used during daily living at the barracks, works, or military maneuvers and training due to the absence of basic communication tools, thus providing great benefits for the national military defense [6].

It can be used as a tool for supplementing the absence of basic communication tools for soldiers, thereby easing the reliance of the communication tool on the levels of soldiers' skills. It could also greatly improve the inefficiency associated with the primitive communication tools used in work or training, thus raising the efficiency of military human resource use. In addition, the low-power consumption characteristics of Zigbee can reduce the costs of repairing and maintaining the communication network and provide great advantages in maintaining smooth communication during wartime. The low data transfer rate of Zigbee can reduce the damage caused by the security problem more than the other communication methods exhibiting a higher transfer rate. It also reduces the merit of using a communication network for the individual soldier's personal purpose.

Fig. 3 shows OPNET-based Squad's Zigbee Local Network Configuration.

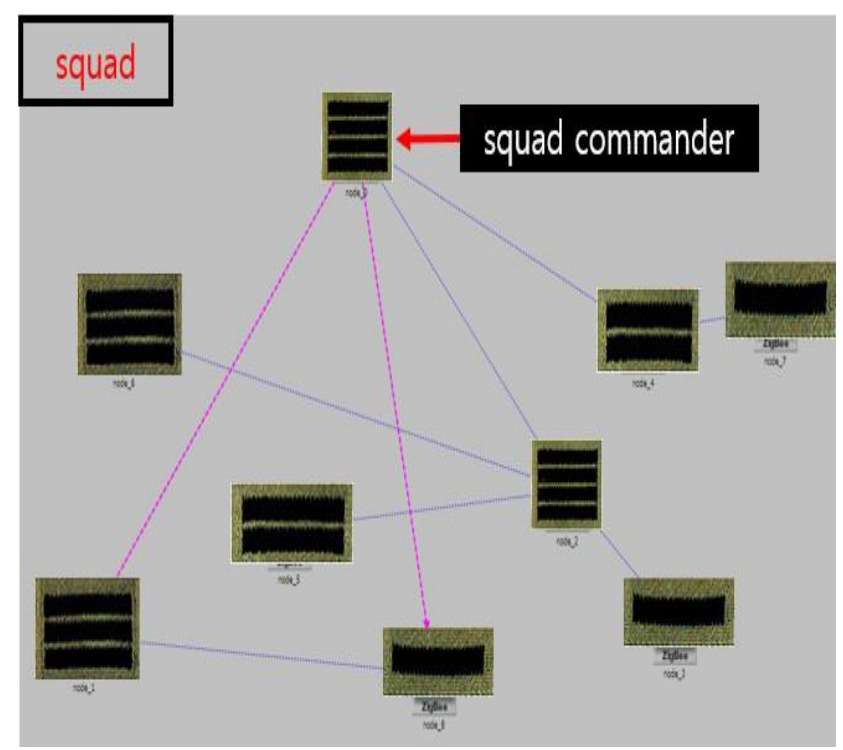

Fig. 3. OPNET-Based Squad's Zigbee Local Network Configuration.

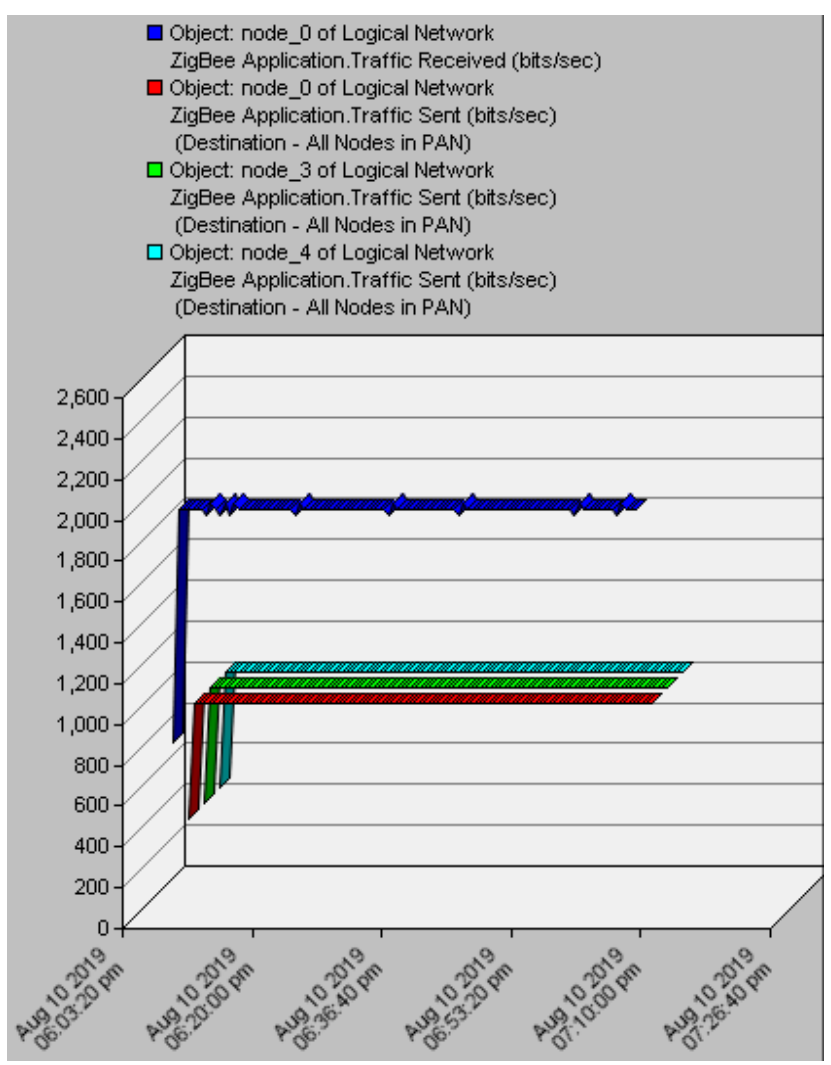

Fig. 4. OPNET-Based Squad's Zigbee Local Network Data Tx/Rx

To achieve Zigbee-based communications, communication distance should be maintained within the allowable range which can be flexible depending on the placement of each node. When operating a tactical squad, the distance between soldiers is determined by the commanding officer so that the communication distance can be variable depending on the arrangement of soldiers, which the officer should be well-acquited with and control his/her troops.

It is necessary to process and supply the node devices which allow Zigbee communications in a form suitable for the soldiers to carry and use in a tactical situation. Additionally, as the communication distance or availability are determined by the commanding officers, it is also necessary to provide a tactical operation education to them. At the same time, soldiers should also acquire basic knowledge about equipment operation to improve utilization of node devices. As such knowledge is easier to acquire than the knowledge needed for some of the primitive communication methods and contributes to the enhancement of tactical performance, a Zigbee communication-based network is well worth for the military use. 
In addition, the mesh network supported by Zigbee does not require any additional efforts or resources for network configurations, thus enabling easy network configuration. Besides the cost advantages, it can improve the time and spatial limitations for network configuration at night and reduce the manpower required for specific tasks. Even when the military manpower is divided during strategic operations, it is still possible to form a local network to maintain the command and control system of a squad base. In addition, soldiers at the rear end who have a basic intuitive understanding of network configuration, and who have received basic training, can easily operate it without having to undergo any difficult training. Here, "Basic intuitive understanding" refers to an individual's understanding of Zigbee's limited communication range. As far as this limitation is addressed, there is no other limitation on network operation, nor is there a need for additional efforts on the part of the soldiers. Fig. 4 shows OPNET-Based Squad's Zigbee Local Network Data Tx/Rx.

\section{OPNET-based Simulation of Zigbee's Distance Limitation}

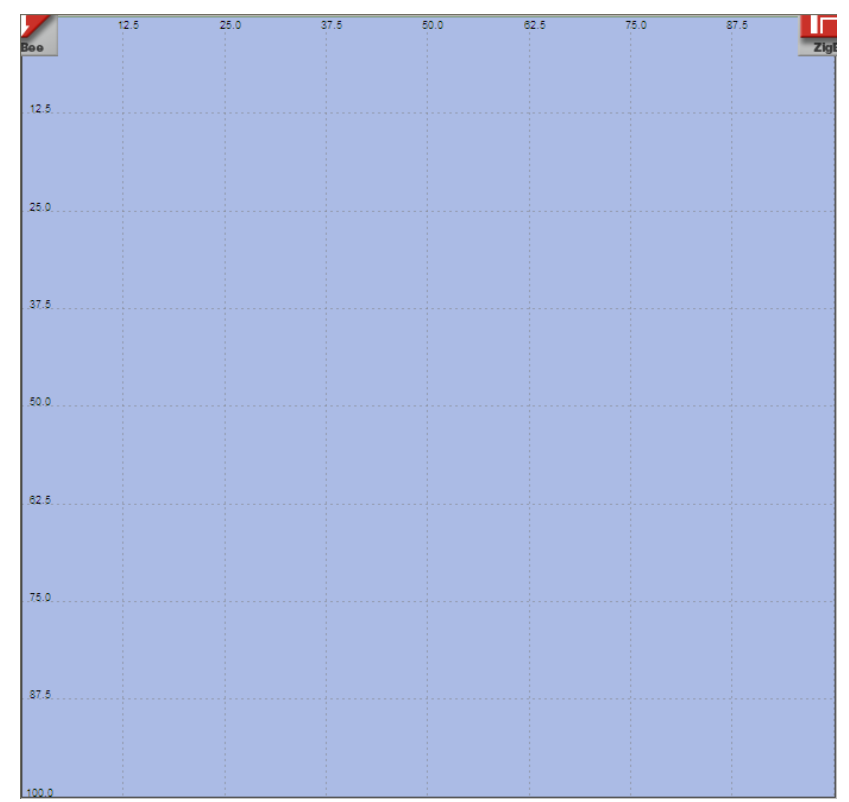

Fig. 5. Distance of $100 \mathrm{~m}$, Office Environment; Communication bet ween Nodes 0 and 1 .

For a distance of $100 \mathrm{~m}$, as shown in Fig. 5, Zigbee assumes communication between multiple nodes; in this simulation, however, in order to simulate explicitly the communication distance of Zigbee without the effects of other restrictions, the number of nodes is limited simply by setting communication between nodes 0 and 1 . In the actual environment, the communication distance can vary accordingly.
As shown in Fig. 6, when all other conditions are the same, if the distance between nodes 0 and 1 is $100 \mathrm{~m}$, smooth communication between the two nodes is confirmed.

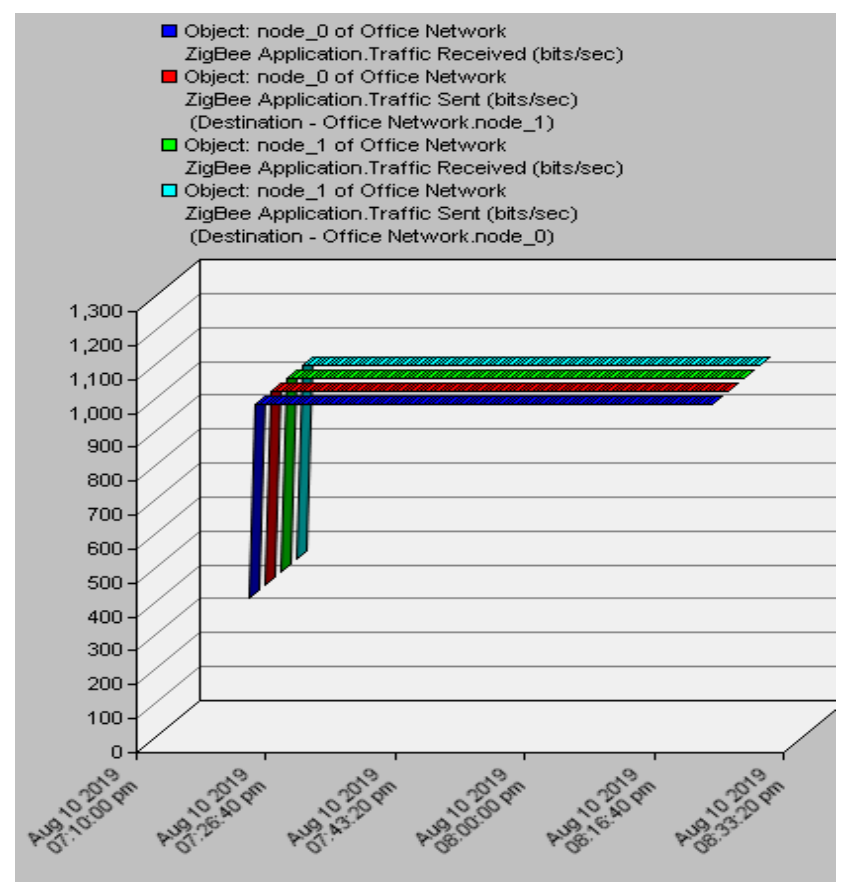

Fig. 6. 100m between Nodes 0 and 1; Office Environment; Data T ransmission.

As shown in Fig. 7, the simulation is performed with nodes 0 and 1 situated about 283 meters apart. All other conditions are the same, and only the location value inside the red box differs. For node 0 , it is at the home location; for node 1 , it is located at the coordinates of $(200,200)$.

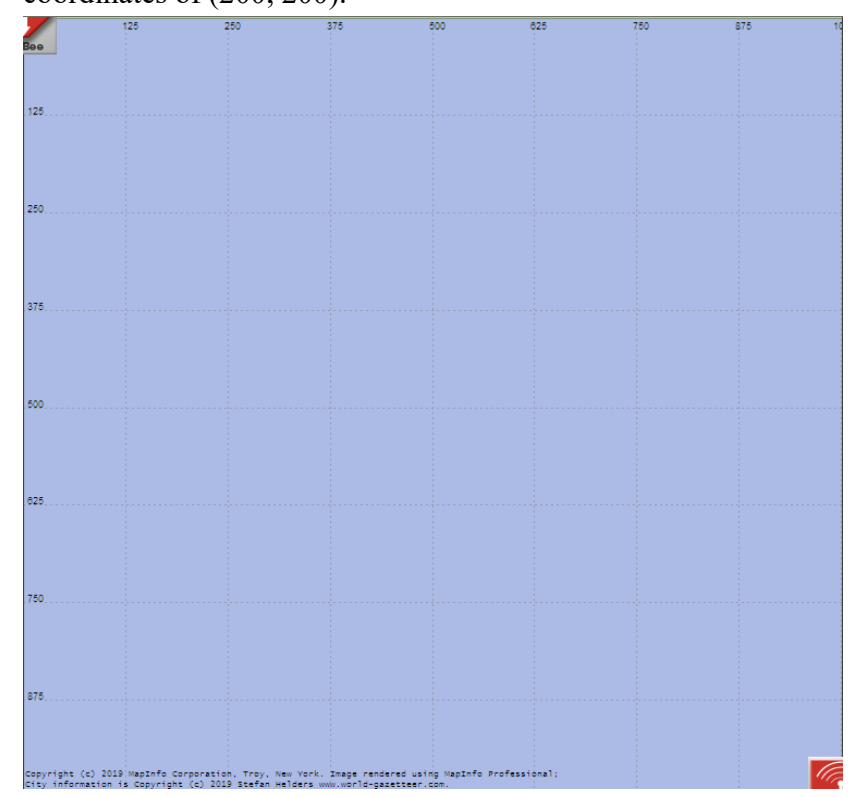

Fig. 7. About $283 \mathrm{~m}$ between Nodes 0 and 1; Office Environment; Communication between Nodes 0 and 1 .

In addition, while all other conditions are the same, if nodes 0 and 1 are situated about 283 meters apart, it is 
simulated that smooth communication is achieved between the two nodes. Fig. 8 shows $283 \mathrm{~m}$ between Nodes 0 and 1 ; Office Environment; Data Transmission.

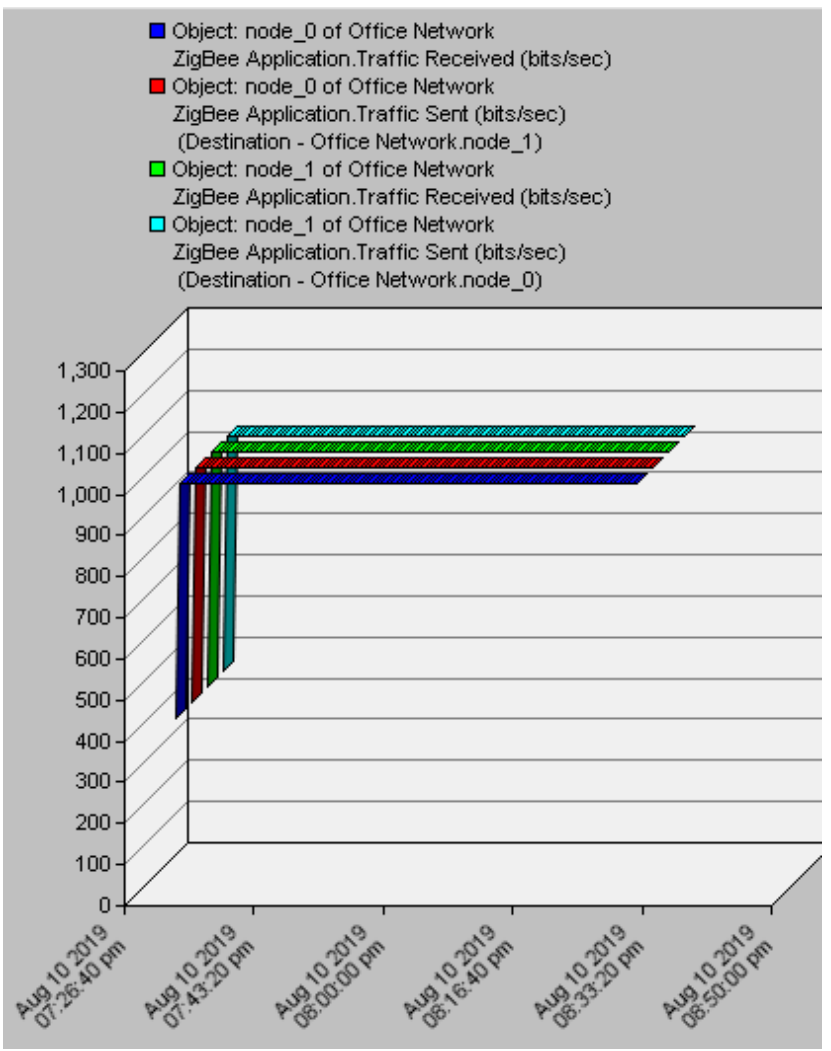

Fig. 8. $283 \mathrm{~m}$ between Nodes 0 and 1; Office Environment; Data T ransmission.

On the other hand, Fig. 9 shows the node setting when nodes 0 and 1 are situated about 1414 meters apart. All other conditions are the same, and only the location value inside the red box differs. For node 0 , it is at the home location; for node 1 , it is located at the coordinates of (1414, 1414).

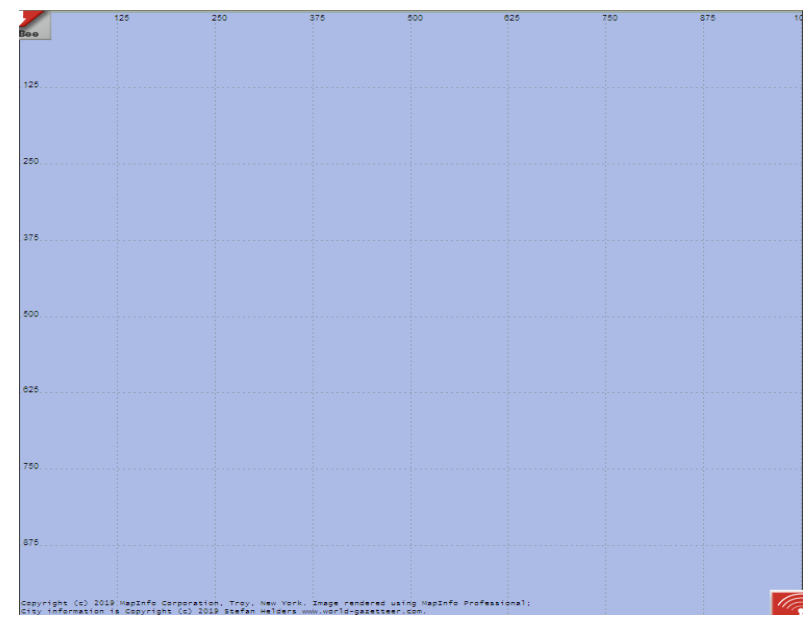

Fig. 9. About $1414 \mathrm{~m}$ between Nodes 0 and 1; Office Environment; Communication between Nodes 0 and 1 .
As shown in Fig. 10, while all other conditions are the same, if nodes 0 and 1 are situated about 1414 meters apart, it is simulated that smooth communication is achieved between the two nodes.

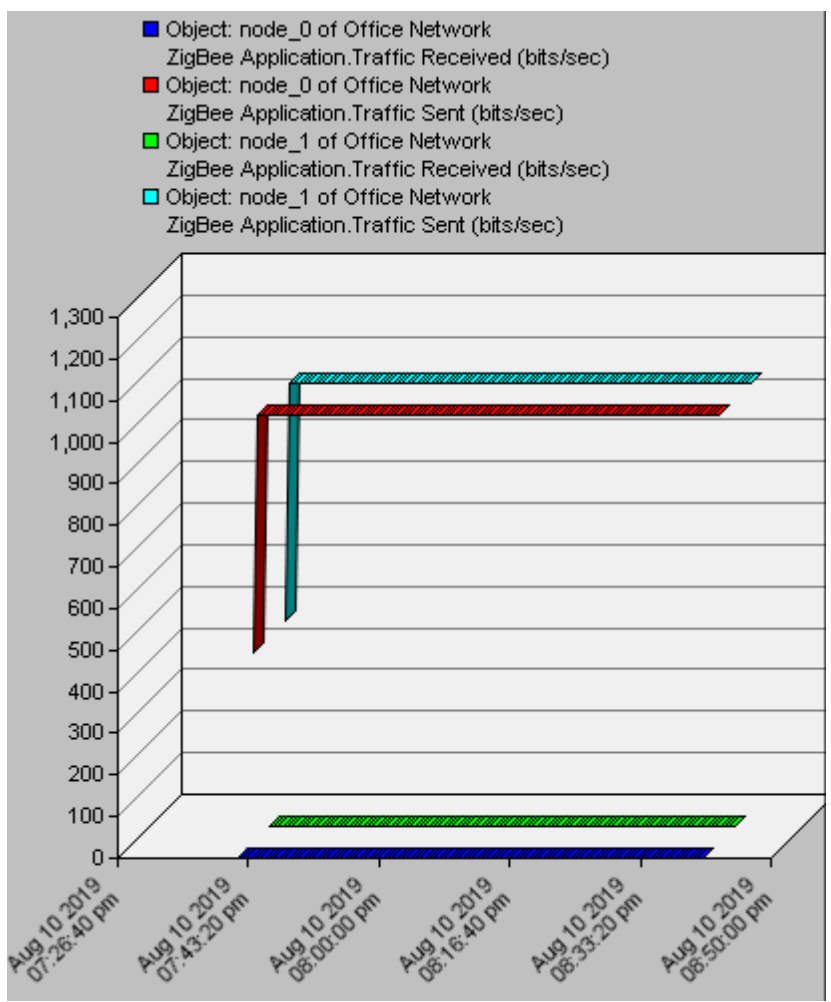

Fig. 10 1414m between Nodes 0 and 1; Office Environment; Data Transmission between Nodes 0 and 1 .

The HRP finds the routing path using the hierarchical addressing scheme based on the parent-child relationship. The HRP requires no broadcast overhead to find the routing path and no memory overhead to maintain routing information. However, the HRP often provides inefficient and fragile routing paths as explained later. [20]

EHRP is an improved form of HRP and able to reset the routing path by finding the shortest hierarchical path when there is a link failure. It is compatible with the Zigbee v.1.0 standard. When configuring a network by using this routing algorithm, it is expected that efficient communications can be achieved in a rapidly changing tactical environment.

\section{CONCLUSIONS}

Zigbee enables flexible connection. Soldiers at the rear end, who have a basic intuitive understanding of network configuration, and who have received basic training only, can operate Zigbee easily. Moreover, no additional costs are incurred for network configuration. For these reasons, it is possible to manufacture and distribute Zigbee communication devices to soldiers at a very low cost. In 
addition, the existing advantages of Zigbee, including its low cost, low power consumption, low data tx speed, and high battery efficiency, make it possible to replace primitive communication tools successfully while maximizing the advantages of its inherent merits.

Thus, it will be possible to overcome the temporal and spatial limitations and ease the reliance of military operations and the efficient use of military manpower on the skill levels of soldiers and achieve a fast and accurate command system. In addition, the expandability of Zigbee could help in implementing a sensor network that can monitor information about individual soldiers, such as their health information, thus improving the efficiency of military manpower use. Furthermore, by processing the big data collected from such a network, they can gain insights from statistics that could help improve the efficient use of soldiers. By using the block chain method, the author believes that it is possible to verify each node of Zigbee beyond the existing security paradigm. The author would like to return to this subject for future studies.

This study discussed the synergy effect arising from combining the merit of a mesh network organically configured based on Zigbee communications in a military tactical network with the troop management at the discretion of the commanding officer but the requirements for data communications bandwidth and protocols were not discussed. These will be dealt with in the future study. And This study supports that the merit of Zigbee communication is appropriate for supplementing the insufficiency in squad unit-level tactical communications. The requirements for this method for actual use in the military will be presented in the future study.

The simulation result showed that the Zigbee-based network configuration could be limited considerably depending on the distance. Nevertheless, when configuring a network, Zigbee has the merits of allowing a fluid change according to the tactical environment and establishment of a wireless network and requiring not much cost for either network configuration or nodes, as well as allowing to divide a single network into two or more networks with a routing algorithm. Also, considering the fact that some of the squad soldiers are still using a primitive method (e.g., waving flags or using hand signals), it is expected that the use of Zigbee communications will improve communication efficiency and increase tactical options.

\section{REFERENCES}

[1] Afanasyev, A., Burke, J., Refaei, T., Wang, L., Zhang, B., \& Zhang, L.; A brief introduction to Named Data Networking. In MILCOM 2018, 2018 IEEE Military Communications Conference (MILCOM) (pp. 1-6).
IEEE, 2018.

[2] Vakilinia, I., Cheung, S., \& Sengupta, S. (2018, October). Sharing susceptible passwords as cyber threat intelligence feed. In MILCOM 2018-2018 IEEE Military Communications Conference (MILCOM) (pp. 1-6). IEEE.

[3] Yen, L. H., \& Tsai, W. T. (2010). The room shortage problem of tree-based ZigBee/IEEE 802.15. 4 wireless networks. Computer Communications, Elsevier, 33(4), 454-462.

[4] Baronti, P., Pillai, P., Chook, V. W., Chessa, S., Gotta, A., \& Hu, Y. F. (2007). Wireless sensor networks: A survey on the state of the art and the 802.15. 4 and ZigBee standards. Computer communications, Elsevier, 30(7), 1655-1695.

[5] Farahani, Shahin., "Zigbee wireless networks and transceivers," Newnes, 2011.

[6] D.J. Watts, S.H. Strogatz., "Collective Dynamics of small-world networks," Nature, Vol. 393, pp. 440-442, June 1998.

[7] J-H Huh., K. Seo., "PLC-Based Smart grid Home Network System Design and Implementation using OPNET Simulation," Journal of Multimedia and Information System, Vol.1, No.2, pp.113-120, 2014.

[8] Huh, J. H.; "PLC-based design of monitoring system for ICT-integrated vertical fish farm," Human-centric Computing and Information Sciences, 7(1), 1-19, 2017.

[9] Status of Military Resources, Military Stats Annual Report.

[10] Marghescu, C., Pantazica, M., Brodeala, A., Svasta, P. (2011, October), "Simulation of a wireless sensor network using OPNET," In 2011 IEEE 17th International Symposium for Design and Technology in Electronic Packaging (SIITME) (pp. 249-252). IEEE.

[11] Huh, J. H., Koh, T., Seo, K.; "Design of a shipboard outside communication network and the test bed using PLC: for the Workers' safety management during shipbuilding process," In Proceedings of the 10th International Conference on Ubiquitous Information Management and Communication (p. 1-6). ACM, 2016.

[12] Hanzálek, Z., Jurcik, P. (2010). "Energy efficient scheduling for cluster-tree wireless sensor networks with time-bounded data flows: Application to IEEE 802.15. 4/ZigBee," IEEE Transactions on Industrial Informatics, 6(3), 438-450.

[13] Li, X., Peng, M., Cai, J., Yi, C., Zhang, H. (2016). OPNET-based modeling and simulation of mobile Zigbee sensor networks. Peer-to-Peer Networking and Applications, 9(2), 414-423.

[14] Chi, H. R., Tsang, K. F., Wu, C. K., \& Hung, F. H. (2016, October). ZigBee based wireless sensor network in smart metering. In IECON 2016-42nd Annual Conference of the IEEE Industrial Electronics Society (pp. 5663-5666). IEEE.

[15] J-H Huh., K Seo., "RUDP design and implementation using OPNET simulation," Computer science and its applications. Springer, 913-919, 2015.

[16] Bayrakdar, M. E., \& Calhan, A. (2017). Improving spectrum handoff utilization for prioritized cognitive radio users by exploiting channel bonding with 
starvation mitigation. AEU-International Journal of Electronics and Communications, Elsevier, 71, 181-191.

[17] J-H Huh., "Reefer container monitoring system using PLC-based communication technology for maritime edge computing," The Journal of Supercomputing, 2019, 1-23.

[18] W-H Choi, J-H Huh., "A Design of Application through Physical Therapy Big Data Analytics," Journal of Multimedia Information System, 5.3, 2018, 171-178.

[19] Ali, I., Hussain, S. S., Tak, A., \& Ustun, T. S. (2017). Communication modeling for differential protection in IEC-61850-based substations. IEEE Transactions on Industry Applications, 54(1), 135-142.

[20] J. Y. Ha, H. S. Park, S. Choi and W. H. Kwon, "EHRP: Enhanced hierarchical routing protocol for zigbee mesh networks ee," in IEEE Communications Letters, vol. 11, no. 12, pp. 1028-1030, December 2007.

[21] Han Seung Choi, et al. "UWB communication terminal for soldiers." Journal of the Institute of Electronics and Information Engineers 50.5 (2013): 50-58.

[22] J.H Huh., T Koh., K Seo. "NMEA2000 ship area network design and test bed experiment using power line communication with the 3-phase 3-line delta connection method," International Journal of Applied Engineering Research, Research India Publications, 10(11), 27789-27797, 2015.

[23] Bittel, Raymond, et al.; "Soldier phone: An innovative approach to wireless multimedia communications," IEEE Military Communications Conference. Proceedings. MILCOM 98. Vol.3. IEEE, 1998.

[24] J. Park et al., Design of the real-time mobile push system for implementation of the shipboard smart working. In Advances in Computer Science and Ubiquitous Computing, Springer, 541-548, 2015.

[25] Kwiatkowski, Marek, Mathew Elliot.; "Using router diffserv mechanisms to implement military QoS." IEEE Military Communications Conference, MILCOM 2003. Vol.2. IEEE, 960-965, 2003.

\section{Author}

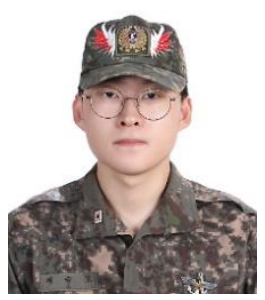

Seung-Mo Je received B.S. in
Engineering Degree from Department of
Computer Engineering, Pukyoug National
University at Daeyeon, Busan, Republic of
Korea in Aug. 2016.
Citation Award by Min-Sik Park,
National Assembly member of the Republic
of Korea (Feb. 11, 2010).

Discharged from 231st Artillery Battalion, 26th Division as a Signal Corpsman, Republic of Korea Army (Oct. 30, 2012-Jul.29, 2014). Citation Award by Hyo-Seob Han, Batallion Commander (Lieutenant Colonel), Information and Communications Batallion, 26th Mechanized Infantry Division, Republic of Korea Army (Jan. 4, 2013).

He received the Best Paper Award from Korea Multimedia Society four times (Oct., 2016 Busan Technopark Outstanding Paper Award,; May., 2017 Best Paper Award,; Oct., 2017; Gyeongsangbukdo Creative Content Agency Outstanding Paper Award,; Oct., 2018 Busan Cinema Center Outstanding Paper Award).
Currently he is Master Candidate at Department of Computer Science Education, Korea University, Republic of Korea. Also, Teacher Training Curriculum (Information and Computer) in accordance with Republic of Korea Secondary Education Act (Aug. 2019). 
Zigbee-based Local Army Strategy Network Configurations for Multimedia Military Service 